\title{
Development of multicellular pollen of Eriobotrya japonica Lindl. through anther culture
}

\author{
María Antonietta Germanà ${ }^{a}$, Benedetta Chiancone ${ }^{a}$, Nathalie Levy Guarda ${ }^{a}$, \\ Pilar S. Testillano ${ }^{\mathrm{b}}$, María-Carmen Risueño ${ }^{\mathrm{b}, *}$ \\ ${ }^{a}$ Dipartimento SENFIMIZO, Facoltà di Agraria, Università degli Studi di Palermo, Viale delle Scienze 11, 90128 Palermo, Italy \\ ${ }^{\mathrm{b}}$ Plant Development and Nuclear Architecture Group, Centro de Investigaciones Biológicas, CSIC, Ramiro de Maeztu 9. 28040-Madrid, Spain \\ Received 28 March 2006; received in revised form 28 June 2006; accepted 5 July 2006 \\ Available online 4 August 2006
}

\begin{abstract}
Eriobotrya japonica Lindl. is a worldwide known tree important for its use as horticultural and ornamental plant, especially in sub-tropical and Mediterranean countries. Microspore embryogenesis through in vitro anther culture is a widely used method to generate genetic variability by obtaining gametic or somatic embryos with many applications for plant breeding. In this work, a protocol has been set up for anther culture in loquat which resulted in the formation of multicellular pollen as a first step to further attempt haploid-plant production via pollen-derived structures. The response of nine of the most widely grown loquat cultivars to anther culture has been evaluated, and four cultivars being selected due to their higher response. The occurrence of anther swelling and the development of calli were analyzed as typical morphological features and potential markers that accompany pollen induction and reprogramming in many systems. Microscopical analysis in responsive anthers and the comparison with the normal gametophytic pollen development was carried out to characterize the cellular changes promoted by the treatment in the anther. The presence of multicellular pollen in the in vitro system developed here indicated the switch of developmental programme which constitutes a crucial step in the design of protocols for the regeneration of microspore-derived embryos and plants. Monitoring structural analysis at different times of the culture revealed specific features of the early microspore embryogenic pathway as well as the cellular organization changes. (C) 2006 Elsevier Ireland Ltd. All rights reserved.
\end{abstract}

Keywords: Microspore embryogenesis; Loquat; Cell structure; Cytochemistry

\section{Introduction}

Eriobotrya japonica originated in China [1] and was introduced to Europe from Japan in the 18th century as an ornamental tree. Later, in the 19th century, selections from cultivars with large fruits were used for fruit production. Loquats are grown mainly in sub-tropical and Mediterranean countries (Spain, Turkey, Italy, Greece and Israel). In fact, this tree has adapted well to the Mediterranean climate and grows in the same areas as citrus species. Spain is the principle country in loquat production in the Mediterranean area, but Italy, particularly Sicily, is also a big producer [2]. Countless trees scattered in small family orchards or gardens are to be seen over the eastern and southern parts of Spain [3]. Fourteen species of

\footnotetext{
* Corresponding author. Tel.: +34 91 8373112; fax: +34 915360432.

E-mail address: risueno@cib.csic.es (M.-C. Risueño).
}

the genus Eriobotrya and more than 100 varieties of E. japonica have been described in China [1,4]. A large number of varieties have emerged in different countries where loquats are grown. Main varieties are selected as seedling variations resulting from natural hybridization. Several studies have described characteristics of promising loquat cultivars obtained by selection from natural variation or breeding [5-9] and a dated study on floral biology has been carried out [10].

Not very much attention has been paid to the use of biotechnology as a tool for obtaining new variability. Biotechnological methods can improve the efficiency and increase the speedy of breeding. Anther culture is a widely used method to generate genetic variability. In fact, through this method, it is possible to regenerate gametic or somatic embryos with many applications for plant breeding. Somatic embryos, for example, can be used for protoplast fusion, genetic transformation or in vitro germplasm storage. Gametic and haploid regenerants are also very important in 
breeding because the single set of chromosomes allows the isolation of mutants and the production of double-haploids, through chromosome doubling. In fact, particularly in woody plants, with a long reproductive cycle, a high heterozygous level and sometimes self-incompatibility, methods for obtaining homozygous plants are of high interest. In fact, haploidization through conventional methods requires several generations of selfing that are not possible to realize in woody plants. Gametic embryogenesis by anther and isolated microspore culture, allows the single-step development of complete homozygous lines from heterozygous parents.

In this work, the response of nine of the most widely grown loquat cultivars to anther culture has been evaluated as a first step to further attempt haploid-plant production via pollenderived structures. The occurrence of anther swelling and the development of calli were characterized and used as typical morphological features and potential markers that accompany pollen induction and reprogramming in many systems. Four cultivars were selected as showing higher response than the rest. Microscopical analysis was carried out in responsive anthers to characterize the cellular changes promoted by the treatments in the anther, as well as to identify the formation of pollen-derived multicellular structures. The comparison with the normal gametophytic pollen development in loquat was also accomplished.

Results showed the formation of multicellular pollen of some loquat varieties, indicating that pollen reprogramming was induced and the first step to set up a protocol of anther culture to induce microspore embryogenesis has been accomplished in loquat. Further work will be necessary to induce differentiation and organogenesis processes to obtain microspore-derived embryos and plantlets.

\section{Material and methods}

\subsection{Plant material and anther culture}

Flower buds were collected from 10-year-old trees of Eriobotrya japonica, cv Algerie, BRT (Baratta) 20, El Buenet, Magdall, Marchetto, Peluche, Sanfilippara, Tanaka and Virticchiara in October 2003 and cv El Buenet, Marchetto, Peluche and Sanfilippara in October 2004. In the first year, for each cultivar, three panicles were selected, their length was measured and the number of flowers counted to characterize the morphology of the panicles of the different genotypes. In the second year, only the four cultivars better responding to anther culture have been selected for the experiment. To check the pollen developmental stage, one anther per each flower size was selected and microspores were stained by $4^{\prime}, 6$-diamidino-2phenylindole dihydrochloride (DAPI, SIGMA) solution $\left(1 \mathrm{mg} \mathrm{ml}^{-1}\right)$ and observed under a fluorescent microscope. Only flower buds of the same size of the ones containing uninucleate microspores were selected for anther culture.

After a 14-day cold pretreatment at $4{ }^{\circ} \mathrm{C}$, flower buds were sterilized by immersion for $5 \mathrm{~min}$ in $70 \%$ (v/v) ethanol, followed by immersion in sodium hypochlorite solution (about $0.5 \%$ active chlorine in water) for $20 \mathrm{~min}$, and finally, they were rinsed three times with sterile distilled water. Petals were aseptically removed with small forceps and anthers carefully dissected and placed in $6 \mathrm{~cm}$ diameter Petri dishes containing $10 \mathrm{ml}$ solid medium. About 50 anthers were placed in each dish and, for each cultivar, 15 Petri dishes were prepared. Petri dishes were sealed with parafilm, incubated at $27+1{ }^{\circ} \mathrm{C}$, for 15 days in the dark, and then placed under cool white fluorescent lamps (Philips TLM 30W/84, France) with a photosynthetic photon flux density of $35 \mu \mathrm{mol} \mathrm{m} \mathrm{m}^{-2}$ and a photoperiod of 16 light hours. Anthers at different culture times: just collected from the tree (time zero), after 3 and 6 months of culture, were selected and fixed for microscopical analysis. Every month up to 10 months after induction, to monitoring the development of anthers in culture, the number of anthers that had not developed or were swollen (with increased size, but identical shape of the anthers just placed in culture) or that had produced calli were observed in each Petri dish. In the second year of culture, the number of open anthers and of anthers surrounded by germinated pollen grains were scored. These values were used to calculate means. In the first year of culture, data were not normally distributed, for this reason a non-parametric test, ANOVA on ranks, was applied. Differences among means were tested by Dunn's test, at $p<0.05$. In the second year of culture, data were normally distributed, therefore the differences among cultivars on the anther development were tested by analysis of variance (ANOVA). Differences among means were tested by Tuckey Multiple Comparison Test, a parametric test, at $p<0.05$. One experiment was conducted during each year.

\subsection{Media composition}

The basal medium used was N6 Chu [11], supplemented with Murashige and Skoog (MS) vitamins [12], lactose, galactose, L-glutamine, biotin and a combination of growth regulators, added to the culture medium before autoclaving [13]. The $\mathrm{pH}$ was adjusted to 5.8 with $1 \mathrm{~N} \mathrm{KOH}$ before autoclaving $\left(20 \mathrm{~min}, 120^{\circ} \mathrm{C}\right)$. Agar (from Sigma) was supplemented as gelling agent.

\subsection{DAPI staining}

For microscopical observation, anthers taken from culture were immersed for $10 \mathrm{~min}$ in $70 \%$ ethanol, rinsed with distilled water and then squashed on a glass slide with some drops of DAPI solution $(1 \mathrm{mg} / \mathrm{ml})$.

\subsection{Cryoprocessing for light microscopy}

Samples were fixed in $4 \%$ paraformaldehyde in phosphate buffered saline (PBS), $\mathrm{pH} 7.3$, overnight at $4{ }^{\circ} \mathrm{C}$. After washing in PBS, samples were dehydrated through a methanol series by progressing lowering of temperature (PLT), from 0 to $-30{ }^{\circ} \mathrm{C}$. Then, the samples were infiltrated and embedded in Lowicryl $\mathrm{K} 4 \mathrm{M}$ resin at $-30{ }^{\circ} \mathrm{C}$ under UV irradiation. Semi-thin sections were obtained and contrasted with toluidine blue for light microscopy analysis. 


\subsection{Cytochemical staining of starch}

One to two micrometers semithin Lowicryl sections were stained with $2 \%$ iodide-potasium-iodide $\left(\mathrm{I}_{2} \mathrm{KI}\right)$ to preferentially stain starch [14].

\section{Results and discussion}

\subsection{Inflorescence characterization}

A previous analysis of the anatomical characteristics of the inflorescences in each cultivar was conducted in order to establish a clear morphological criterion for the selection of anthers at the responsive developmental stage for culture.

To morphologically characterize the different cultivars, panicle length was measured and the number of flowers was counted per inflorescence. Results are reported in Table 1. Magdall inflorescences were the shortest $(9.0 \mathrm{~cm})$, while the longest were the Algerie ones $(16.2 \mathrm{~cm})$. The cultivar Marchetto showed the highest number of flowers per inflorescence (195), Tanaka and El Buenet the lowest ones (110).

\subsection{Anther culture}

Different features have been described in anther cultures as signs of the initiation of a morphogenic response and the change of the developmental pathway. The swelling of anthers have been described in different systems as the first anatomical change accompanying the morphogenic response, however, the non-responsive anthers decreased in volume and turgescence.

In the first experimental year, anthers from nine different loquat cultivars were in vitro cultured. After 1 month in culture, all the cultivars tested showed that most of the anthers appeared swollen (Fig. 1b), in comparison with the anther size at the beginning of the culture (Fig. 1a), indicating a good response to culture, and only in a few cultivars (Peluche, Sanfilippara, and Marchetto) anthers produced calli at this early time point. Callus production is desiderable because it is possible to obtain, starting from it, regeneration through indirect embryogenesis and plantlet production. After 10 months in culture, no substantial difference was observed in the percentage of the swollen anthers (Fig. 1c), while anthers with callus of the cultivars Peluche and Sanfilippara, significantly increased.

Table 1

Inflorescence length and number of flowers per inflorescence

\begin{tabular}{lcl}
\hline Cultivar & Inflorescence length $(\mathrm{cm})$ & $\begin{array}{l}\text { Number of flowers } \\
\text { per inflorescence }\end{array}$ \\
\hline Algerie & 16.2 & 164 \\
BRT 20 & 12.6 & 138 \\
El Buenet & 13.6 & 110 \\
Magdall & 9.0 & 156 \\
Marchetto & 10.1 & 195 \\
Peluche & 12.3 & 155 \\
Sanfilippara & 9.4 & 115 \\
Tanaka & 12.3 & 110 \\
Virticchiara & 16.0 & 152 \\
\hline
\end{tabular}

Anther of the cultivar El Buenet started to produce calli (Fig. 2b) only after 8 months in culture. Statistical analysis showed significant differences among the cultivars regarding the swollen anthers as well as the anthers with calli. El Buenet had the statistically lowest percentage of swollen anthers (59.7\%) (Fig. 1c); while Peluche and Sanfilippara were the cultivar with the highest percentage of anthers with callus (respectively 3.8 and 3.5\%) (Fig. 2d). Marchetto, Algerie, Virticchiara and BRT 20 showed the dispersion and germination of pollen grains in the medium that indicated that microspores followed the gametophytic pathway (Fig. 2a).

In the second year of experiments, anthers only from the four selected cultivars showing significant percentages of calli production (Peluche, Sanfilipara, Marchetto and El Buenet) were put in culture and analyzed. The occurrence of both features, swollen anthers and calli production, was again tested for the selected cultivars and statistical analysis showed significant differences among them. Peluche was the cultivar with the statistically lowest percentage of swollen anthers (53.2\%) (Fig. 1d). Regarding the anthers with calli, the four selected cultivars exhibited higher percentages than the previous year, being up to $20 \%$ in all cases and showing the Peluche cultivar the highest proportion of anthers with calli (38.5\%) (Fig. 2e). Moreover, in Peluche and Marchetto, the highest percentage of open anthers (Fig. 2c) was observed (respectively 8.2 and $6.9 \%$, data not shown).

The results obtained showed that the response of loquat anthers to in vitro culture was different depending on the year; as for many trees, this different response could be probably associated with the yearly-changing weather conditions affecting the proportion of high-quality pollen formation in the anthers. Thus, Peluche, for example, registered $3.8 \%$ of anthers with callus in the first year of experiments and $38.5 \%$ in the second one. Moreover, as previously observed in other species, such as Citrus [15], the response of loquat anther to in vitro culture was genotype-dependent, meaning that different results have been obtained from different cultivars of the same species during the same experiment. Results indicated a better response in four of the nine cultivars studied.

\subsection{Cellular architecture}

In order to characterize the main changes in the cellular architecture of microspores and pollen grains promoted by the treatments in the anther, as well as to identify the formation of pollen-derived multicellular structures, a microscopical analysis was carried out in the responsive cultivars. Anthers directly excised from the tree (time zero) and anthers collected at various culture times were fixed, processed and analyzed under light and fluorescence microscopy. The results showed that the processing method selected, using formaldehyde fixation, low temperature dehydration and embedding in Lowicryl resin, was adequate for a good structural preservation of loquat anthers in culture. The selection of the processing method is a key point for structural studies, especially in the case of woody species $[15,16]$. Formaldehyde fixation, low temperature processing and Lowicryl resin embedding have shown to be convenient for 

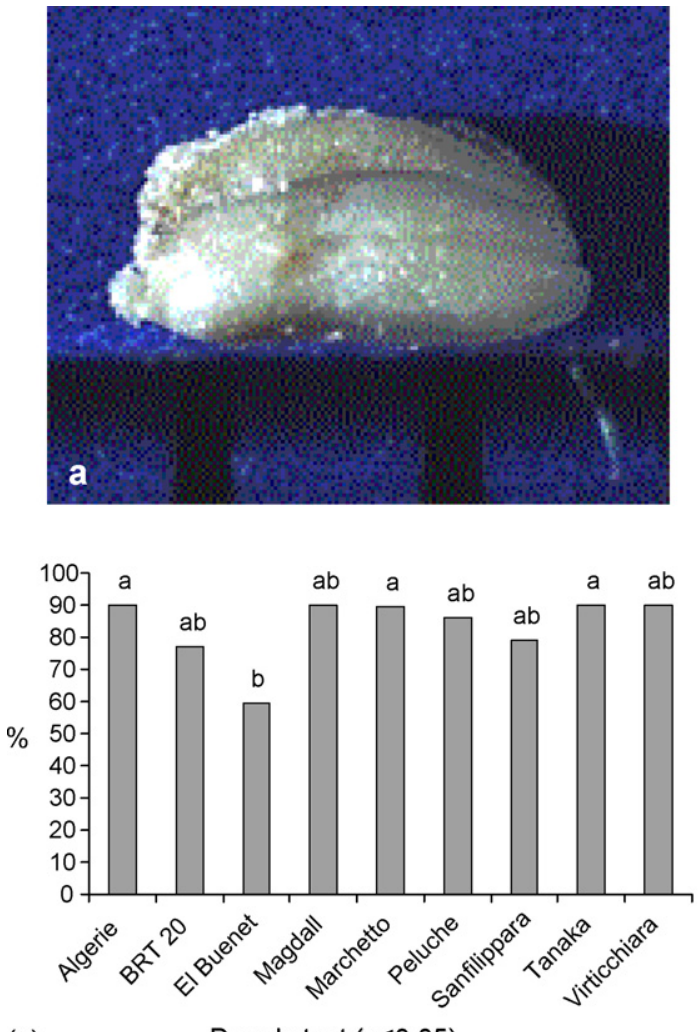

(c)

Dunn's test $(p \leq 0,05)$
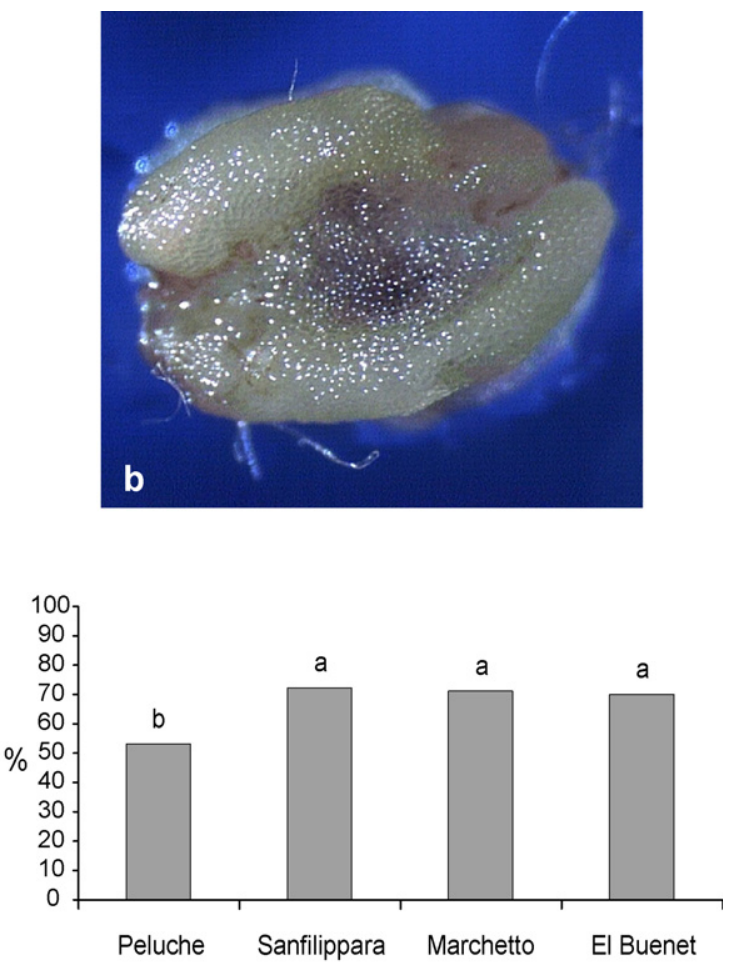

(d)

Tukey test $(p \leq 0,05)$

Fig. 1. Loquat anther culture. (a) Anther at time zero. (b) Swollen anther of cultivar Tanaka after 10 months in culture. (c) Percentages of swollen anthers after 10 months in culture in 2003. (d) Percentages of swollen anthers after 8 months in culture in 2004.
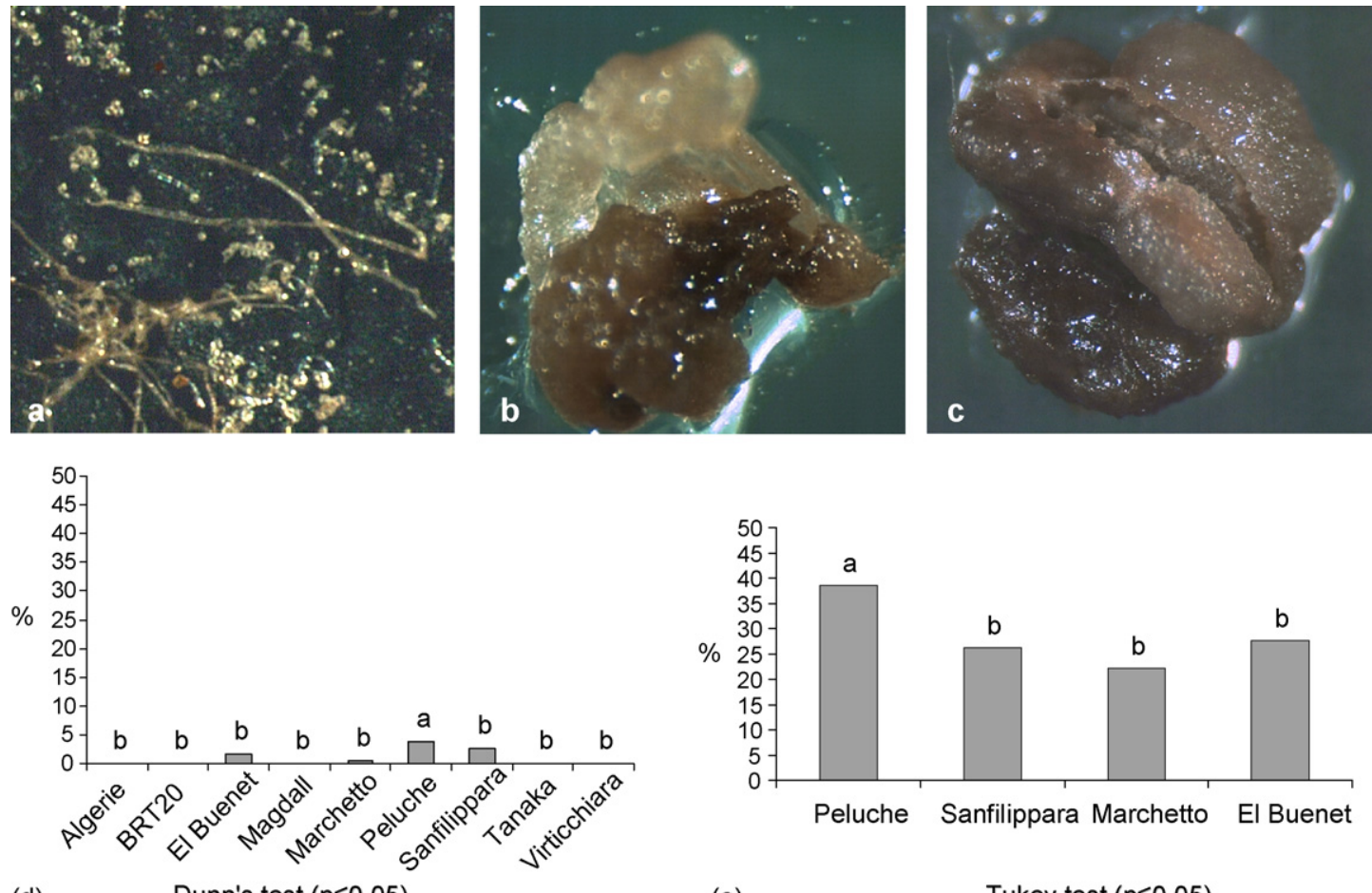

(d)

Dunn's test $(p \leq 0,05)$

(e)

Tukey test $(p \leq 0,05)$

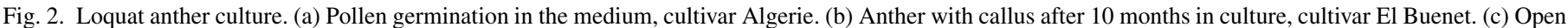

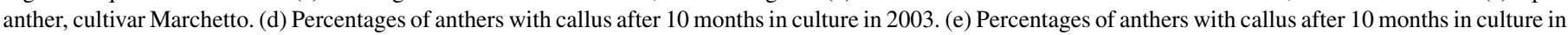
2004. 

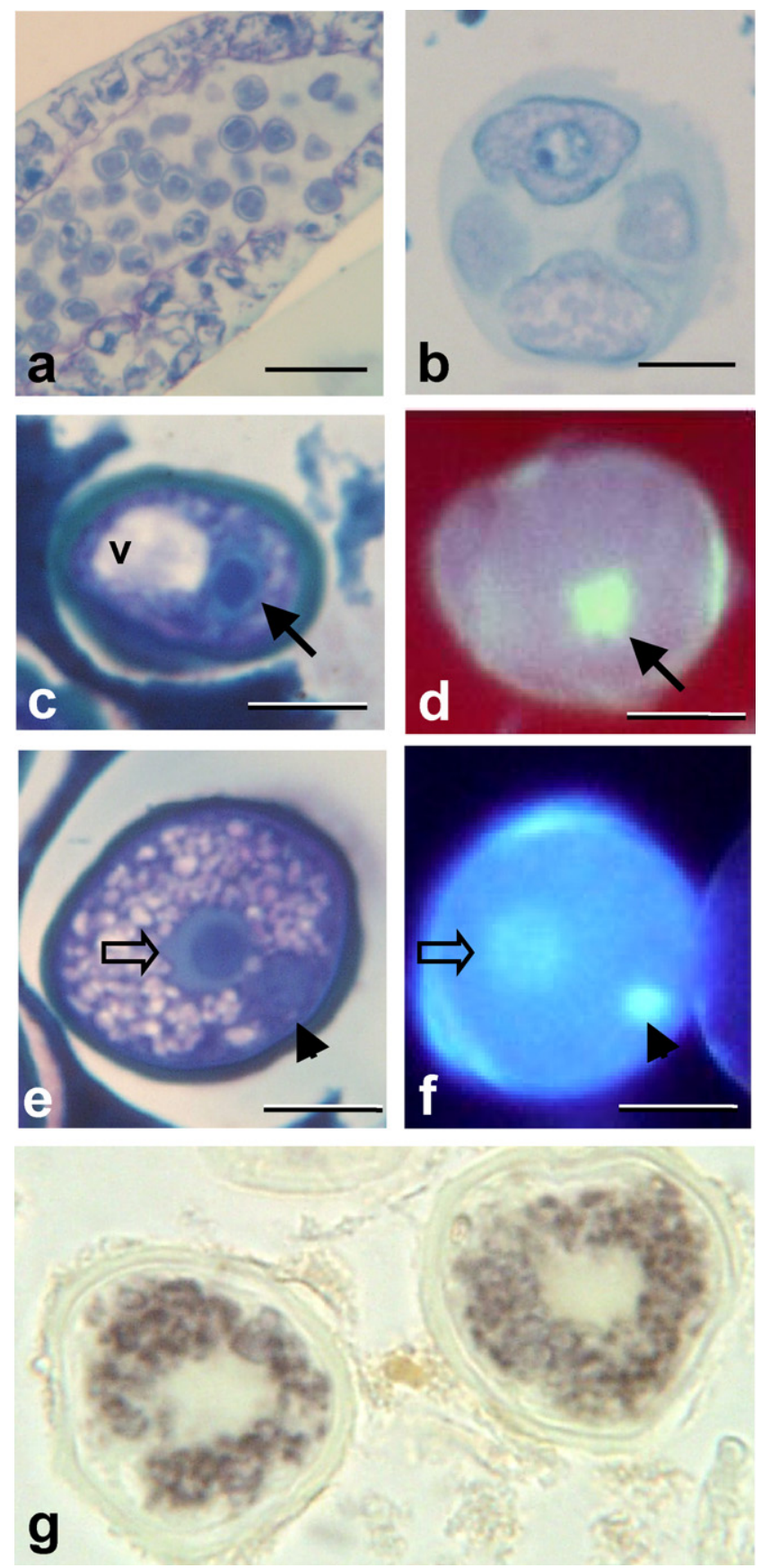

Fig. 3. Cellular organization of microspores in anthers at time zero. Different stages of the microspore gametophytic development. Semithin Lowicryl sections, toluidine blue staining for general structure (a, b, c and e), DAPI staining for DNA ( $d$ and $\mathrm{f}$ ), and $\mathrm{I}_{2} \mathrm{KI}$ cytochemistry for starch (g). (a) Panoramic view of the anther containing microspores in the tetrade stage. (b) High magnification of a tetrade of microspores. (c and d) Vacuolate microspore showing the large vacuole (v), and the nucleus (arrow) with intense DAPI fluorescence (d) located at the periphery. (e and f) Young bicellular pollen with dense cytoplasms containing abundant clear inclusions (e), the large vegetative nucleus (open arrow) shows faint DAPI fluorescence in contrast with the generative one (arrow) located at the periphery and smaller, exhibiting higher fluorescence after DAPI staining (f). (g) Starch cytochemistry on bicellular pollen grains, numerous starch grains are revealed in the cytoplasms as dark deposits. Bars in (a): $50 \mu \mathrm{m}$, in (b-f): $10 \mu \mathrm{m}$. structural analysis of anther culture development in several trees, such as Quercus [16,17], Citrus [18], and in this work, for the first time, in Eriobotrya.

Anthers of different sizes were collected from the trees and analyzed to characterize the main stages of pollen development in loquat (Fig. 3), which displayed similar cellular organization in the cultivars studied. Anthers collected from the tree (time zero) showed various cell layers of somatic
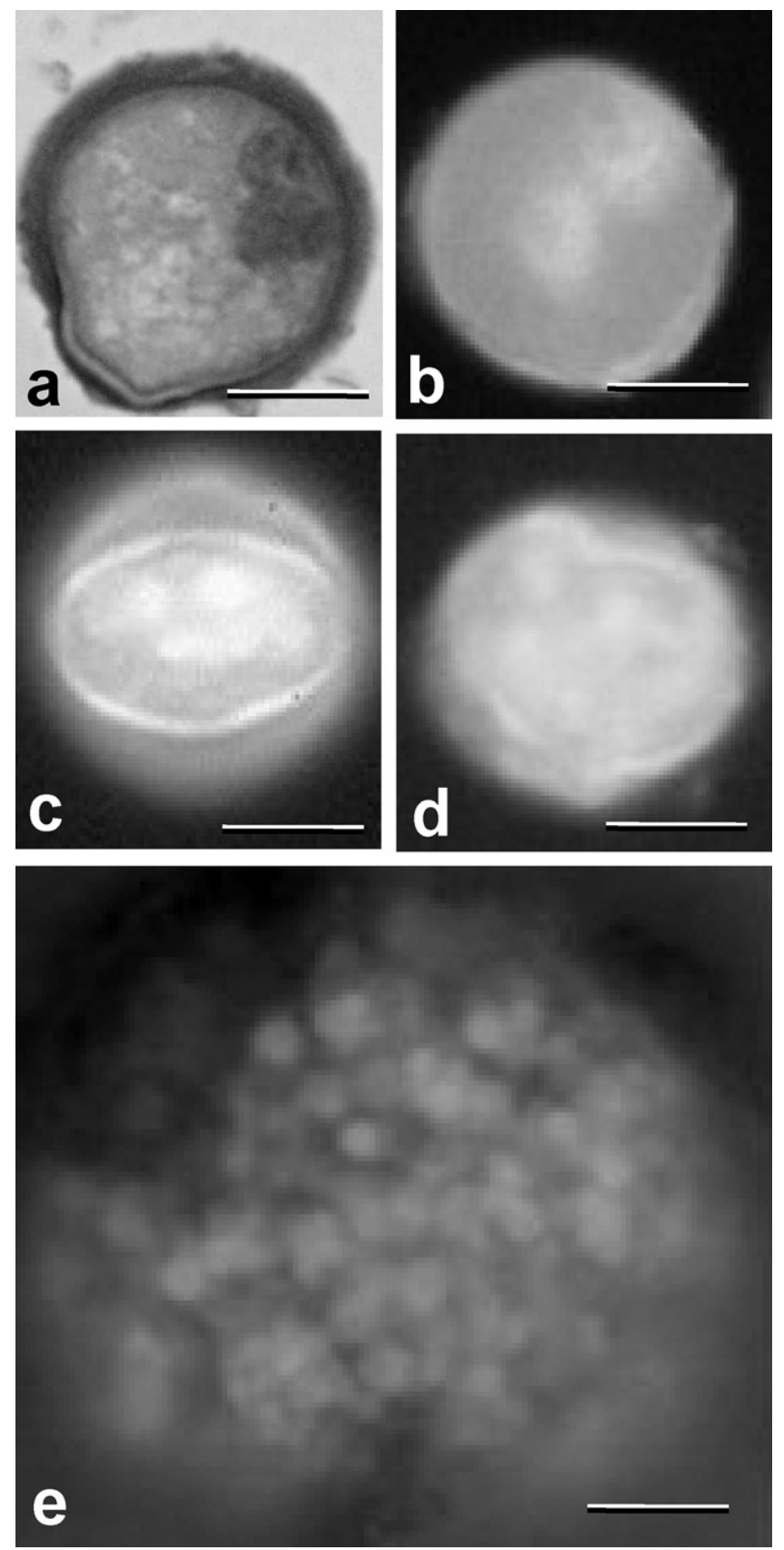

Fig. 4. Multicellular structures originated during the anther culture. DAPI staining for DNA in squashed preparations of anthers at different culture times (b-e), and semithin sections stained with toluidine blue for general structure (a). (a and b) Pollen grain with two nuclei showing similar size, shape and fluorescence intensity after DAPI (b) originated by a symmetrical division. (c and d) Multicellular pollen grains still surrounded by the exine. (e) Large multicellular proembryo after the exine breakdown showing a rounded-elongated shape with numerous small nuclei. Bars represent $20 \mu \mathrm{m}$. 
tissue with the typical features of exotecium, intermediate layer and endotecium (Fig. 3a). The youngest microspore stage found in the anthers at time zero was tetrade (Fig. 3a and b), showing a large and rounded central nucleus in each cell and a thick callose wall (Fig. 3b). Most of the anthers collected at time zero mainly contained microspores at a later developmental stage, e.g. vacuolated microspores (Fig. 3c and $\mathrm{d}$ ) and, very rarely bicellular pollen (Fig. 3e and f). The vacuolated microspores showed a large cytoplasmic vacuole and a peripheral nucleus with a large nucleolus (Fig. 3c and d); a thick exine was also observed in the semithin sections (Fig. 3c). The bicellular pollen contained nuclei with different chromatin condensation pattern, as seen by the fluorescence brightness after DAPI staining. The vegetative nucleus appeared larger and showed a faint DAPI fluorescence while the generative one was smaller and very brilliant due to the high condensation of its chromatin (Fig. 3f). The vegetative cytoplasm showed many clear spots (Fig. 3e), and after the iodide-based staining for starch, these inclusions appeared dark (Fig. 3g), revealing a high starch accumulation in the bicellular pollen grain.
In contrast, anthers in culture contained pollen with differential features. After 3 and 6 months in culture, selected anthers were DAPI stained and squashed for checking the number of nuclei in the pollen grains and derived structures. Results are shown in Fig. 4. After 3 months in culture, pollen grains with two or several nuclei were observed (Fig. 4a-d); in some cases pollen grains with two similar nuclei (Fig. 4a and b), formed by a symmetrical division, were found, indicating a high asynchrony in the embryogenic response of the microspores In some anthers, after 6 months in culture, large multicellular structures were observed (Fig. 4e), which were rounded or elongated and did not show the exine at their periphery. These observations indicated that microspores switched their developmental programme towards proliferation, giving rise to multicellular pollen grains through the in vitro culture protocol developed in this work for loquat anthers. The results presented here suggest that the vacuolate microspore, very abundant in most of the anthers at the beginning of culture, was a responsive stage for induction in loquat, as reported in many other herbaceous $[14,19,20]$ and woody species $[17,18]$.

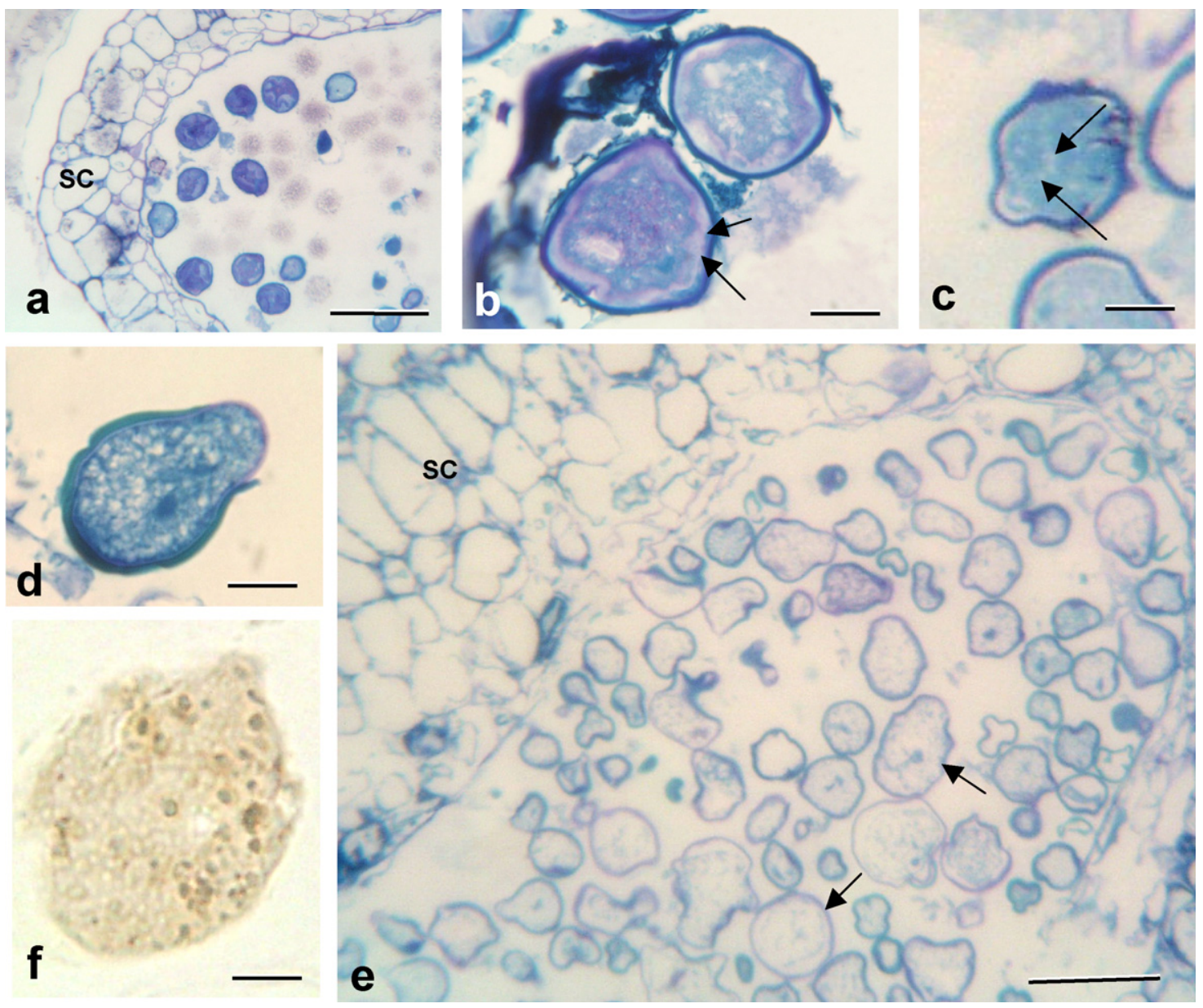

Fig. 5. Cellular organization of multicellular structures in anthers at different culture times after induction. Semithin Lowicryl sections, toluidine blue staining for general structure (a-e), and $\mathrm{I}_{2} \mathrm{KI}$ cytochemistry for starch (f). (a-c): Multicellular pollen grains in anthers after 3 months in culture. They show dense cytoplasms and a thick and clear cell wall underneath the exine (small arrows). Inner cell walls (arrowheads) can be seen in some pollen grains (c). (d-f) Structural organization of an anther after 6 months in culture (e) showing some large rounded and elongated multicellular structures (arrows), some of them with the exine broken (d) and numerous abnormal pollen grains inside the pollen sac. (f): Starch cytochemistry revealing dark deposits in these proembryos. SC: somatic cells of the anther wall. Bars in (a and e): $50 \mu \mathrm{m}$, in (b-d and f): $20 \mu \mathrm{m}$. 
The structural organizations of these multicellular pollen grains were analyzed on semithin sections (Fig. 5). After 3 months in culture, multicellular pollen grains, still surrounded by the exine, appeared as very dense individual structures localized inside the swollen area of the pollen sac (Fig. 5a). They showed a similar structure than multicellular microsporederived structures or proembryos found in other woody (17) and herbaceous (14) species. In these anthers and in anthers after 6 months of culture, the covering layers of the pollen sac have changed with respect to non-cultured anthers. They showed an increased number of cell layers with polygonal cells and similar features among the modified layers (Figs. 3a and 5a-e). The multicellular structures displayed dense cytoplasms and a thick cell wall below the exine which appeared as an unusual clear layer after toluidine blue staining (Fig. $5 \mathrm{~b}$ and c). In some of them, the presence of inner cell walls could be distinguished (Fig. 5c, arrows). At early stages of microspore embryogenesis, multicellular pollen grains were reported displaying small cells with large rounded nuclei and dense cytoplasms, as typical features of proliferating plant cells $[14,21,22]$, showing very different structural organization from that of normally developing pollen grains. The presence of a thick inner wall underneath the exine has been reported as a characteristic of the multicellular structures formed after embryogenesis induction on various in vitro systems $[14,16,17]$, constituting a possible marker of the new developmental pathway. At later stages, elongated structures bursting out of the exine could be observed (Fig. 5d). After 6 months in culture, larger multicellular structures appeared in the interior of the anther displaying less dense cytoplasms (Fig. 5e and f, arrows). These multicellular structures showed rounded or elongated shapes in section, and contained starch deposits, as revealed by the IIK cytochemistry (Fig. 5f). Inside the anther, some abnormal pollen grains of heterogeneous shapes and sizes were also observed. The somatic tissue of the anther wall appeared unusually thick, with additional cell layers (compare Fig. 5a and e). The accumulation of starch at the early stages of microspore embryogenesis has also been demonstrated in Citrus, by specific cytochemistry [18], and suggested in anther culture of Quercus [17]. In other systems of herbaceous species, starch deposits were also found at later stages, in multicellular proembryos, being associated to the initiation of differentiation [14].

\section{Conclusions}

The regeneration of microspore-derived embryos and plants are very important goals in the breeding of woody plants and particularly of loquat that has not been much studied yet with a biotechnological approach. In this work, a protocol for in vitro anther culture in loquat has been set up, and its efficiency for inducing multicellular pollen grains has been observed in several cultivars, as a first step to further attempt haploid-plant production via pollen-derived structures. Four cultivars were selected for showing higher response than the rest, the cultivar Peluche giving the best performances in both the years of the experiments. Microscopical analysis of responsive anthers and the comparison with the normal gametophytic pollen devel- opment in loquat revealed the main cellular changes promoted by the treatments and identified the formation of pollen-derived multicellular structures, indicating that pollen reprogramming was induced. The in vitro system described here constitutes the first step to set up a protocol of anther culture to induce microspore embryogenesis in loquat. Further work will be necessary to induce differentiation and organogenesis processes, and to obtain regeneration of microspore-derived embryos and plantlets. Moreover, the present work could provide new insights into the key processes involved in the switch and progression of the microspore from a gametophytic pathway of development to the embryogenic one in woody plants, in which there are only very few information.

\section{Acknowledgements}

This work is a collaboration between the CSIC (Spain) and the Università degli Studi di Palermo (Italy) in the frame of the joint bilateral Spanish-Italian programme of "Acciones Integradas" (HI2002-0099) and "Azione integrata" (IT 929). This work was partially supported by Spanish projects granted by MEC AGL2005-05104 and BFU2005-01094, and by the Italian PRIN 2004.

We thank Giada Solazzo for her technical assistance in loquat anther culture and Ms. Beryl Ligus-Walker for checking the English style.

\section{References}

[1] H.Z. Zhang, S.A. Peng, L.H. Cai, et al., The germplasm resources of the genus Eriobotrya with special reference on the origin of E. japonica Lindl., Plant Breeding Abstr. 63 (1993) 772.

[2] F. Calabrese, Nespolicoltura italiana e spagnola a confronto, Frutticoltura N-1 (1995) 21-25.

[3] Underutilized fruit crops in the Mediterranean region, G. Llácer, U. Aksoy, M. Mars (Eds.), Cahiers Options Méditerranénnes, vol. 13, 1995.

[4] C.K. Ding, Q.F. Chen, T.L. Sun, et al., Germplasm resources and breeding of Eriobotrya japonica Lindl. in China, Acta Horticult. 403 (1995) 121126.

[5] J.S. Huang, X.D. Xu, S.Q. Zheng, New extra-large and early loquat cultivar-Zaozhong 6, China Fruits 4 (1993) 4-6.

[6] R.D. Singh, H. Lal, Studies on ripening, yield and physicochemical composition of some promising loquat cultivars grown at Saharanpur, Horticult. Abstr. 59 (1989) 692.

[7] M.O. Athayde, M.J. Fornazier, A.N. Da Costa, et al., Evaluation of loquat cultivars for the Serrana region of Espiritu Santo, Horticult. Abstr. 62 (1992) 7821.

[8] F. Monastra, O. Insero, Loquat industry in Italy: varieties investigation, Horticult. Abstr. 62 (1992) 3464.

[9] B. Baratta, G. Campisi, A. Raimondo, Miglioramento genetico del nespolo del Giappone (Eriobotrya japonica Linde) cv "Marchetto", Rivista di Frutticoltura e di Ortofloricoltura 57 (1) (1995) 27-32.

[10] F.G. Crescimanno, Ricerche sulla biologia fiorale del Nespolo del Giappone, Riv. della Ortoflorofrutticoltura Italiana 3-4 (1958) 107-120.

[11] C. Chu, The N6 medium and its applications to anther culture of cereal crops, in: Proceedings of Symposium on Plant Tissue Culture, Science Press, Peking, (1978), pp. 43-50.

[12] T. Murashige, F. Skoog, A revised medium for rapid growth and bioassays with tobacco tissue cultures, Physiol. Plant. 15 (1962) 473-497.

[13] M.A. Germanà, B. Chiancone, Improvement of the anther culture protocol in Citrus clementina Hort. ex Tan, Plant Cell Rep. 22 (2003) 181-187. 
[14] I. Barany, P. González-Melendi, J. Mityko, et al., Microspore-derived embryogenesis in Capsicum annuum: subcellular rearrangements through development, Biol. Cell. 97 (2005) 709-722.

[15] M.A. Germanà, Haploidy in Citrus, in: S.M. Jain, S.K. Sopory, R.E. Veilleux (Eds.), In vitro Haploid Production in Higher Plants, vol. 5 , Kluwer Academic Publishers, Dordrecht, The Netherlands, 1997 , pp. 195-217.

[16] C. Ramírez, P.S. Testillano, B. Pintos, et al., Cellular characterization on microspore embryogenesis in anther culture of Quercus suber, in: B. Bohanec (Ed.), Biotechnological Approaches for Utilization of Gametic Cells, Official Publications of the European Communities, Bruxelles, 2001, pp. 247-251.

[17] C. Ramírez, P.S. Testillano, B. Pintos, M.A. Moreno, et al., Changes in pectins and MAPKs related to cell development during early microspore embryogenesis in Quercus suber L., Eur. J. Cell Biol. 83 (2004) 213-225.
[18] C. Ramirez, B. Chiancone, P.S. Testillano, et al., First embryogenic stages of Citrus microspore-derived embryos, Acta Biol. Cracov. Bot. 45 (2003) 53-58.

[19] P. Gonzalez-Melendi, P.S. Testillano, P. Ahmadian, et al., In situ characterization of the late vacuolated microspore as a convenient stage to induce embryogenesis in Capsicum, Protoplasma 187 (1995) 60-71.

[20] J.M. Custers, J.H.G. Cordewener, Y. Nöllen, et al., Temperature controls both gametophytic and embryogenic development in microspore cultures of Brassica napus, Plant Cell Rep. 13 (1994) 267-271.

[21] P.S. Testillano, M.J. Coronado, J.M. Seguí-Simarro, et al., Defined nuclear changes of the microspore accompany its reprogramming to embryogenesis, J. Struct. Biol. 129 (2000) 223-232.

[22] P.S. Testillano, P. González-Melendi, M.J. Coronado, et al., Differentiating plant cells switched to proliferation remodel the functional organization of nuclear domains, Cytogenet. Genome Res. 109 (2005) 166-174. 